\title{
EXCISION OF THE FEMORAL HEAD AND NECK FOR ANKYLOSIS AND ARTHRITIS OF THE HIP
}

\author{
By J. S. BATCheloR, F.R.C.S. \\ Honorary Orthopaedic Surgeon, Guy's Hospital and St. Vincent's Orthopaedic Hospital
}

For many years the tendency has been to regard arthrodesis as the treatment of choice for a stiff and painful hip joint, for when sound bony ankylosis occurs the patient is assured of a strong, stable and painless hip. There are, however, certain disadvantages associated with this procedure. The operation itself may be formidable and the period of immobilization in plaster for three to four months which often follows is not well tolerated by elderly patients. Unless the lumbar spine is supple the fixed hip makes it difficult, if not impossible, for the patient to sit comfortably on an upright chair and to put on shoes and stockings. At a recent orthopaedic meeting a demonstration of devices to enable the patient to dress himself portrayed graphically the difficulties that patients with fixed hips may encounter. Arthrodesis is contra-indicated when both hips are affected or when the lumbar spine is stiff and arthritic.

To produce a new hip joint which is mobile, painless and stable has proved a difficult problem. Formal arthroplasty by remodelling of the femoral head and the insertion of a fascial flap has proved unsuccessful, for although a limited range of movement may be obtained for a short time, the joint soon stiffens and becomes painful. During recent years, however, Smith-Petersen has achieved considerable success with his vitallium cup arthroplasty.

The operation of pseudarthrosis of the hip has made little appeal to the orthopaedic surgeon. The method advocated by Sir Robert Jones, which consists essentially of the excision of a large wedge of bone from the trochanteric region and the attachment of the gluteal insertion to the stump of the femoral neck, is followed by considerable instability and loss of power, for the action of many of the hip muscles, particularly the glutei, is lost.

At a meeting of the British Orthopaedic Association at the Wingfield-Morris Orthopaedic Hospital in 1938 , Professor Girdlestone demonstrated the good results that can be obtained in osteo-arthritis of the hip by excision of the femoral head and neck. This is a simple procedure, well borne by the elderly, which effectively relieves pain and restores movement and leaves undisturbed the attachment of the hip muscles. I have now performed this operation in 34 patients and have found that a free range of painless movement is practically assured.

This procedure has however one serious disadvantage - the potential instability of the pseudarthrosis. This instability, which varies in degree in different hip conditions and depends to a large extent on the amount of fibrosis present around the joint before operation, can be overcome to a certain extent by the use of a calliper for some months after the operation, or more effectively by a low sub-trochanteric osteotomy of the Schanz type. I have therefore supplemented the excision with an osteotomy in a large number of cases and have found that it provides excellent stability. Plating of the osteotomy followed by Hamilton-Russell traction avoids plaster spicas and allows early movement at the new joint.

\section{Glinical Material}

Excision, with or without osteotomy, has been employed in the following conditions :

\section{Number}

of cases

I. Ankylosing spondylitis and rheumatoid arthritis $\quad \ldots \quad \ldots \quad \ldots 7$

2. Traumatic and degenerative osteoarthritis $\quad \ldots \quad \ldots \quad \ldots \quad \ldots 12$

3. (a) Fracture-dislocation of hip $\quad$. 3

(b) Ununited fracture of femoral neck 4

4. Bilateral ankylosis following suppura$\begin{array}{lllll}\text { tive arthritis } \quad \ldots & \ldots & \ldots & 4\end{array}$

5. Chronic suppurative arthritis $\quad . .4$

\section{Indications for Osteotomy}

Osteotomy should be performed in patients with unilateral degenerative and traumatic osteoarthritis, in ankylosing spondylitis and in patients with ununited fracture of the femoral neck and fracture-dislocation of the hip. In these conditions peri-articular fibrosis is minimal and the prolonged use of a calliper cannot be relied upon to give good stability. Osteotomy is particularly indicated when the patient is young and active, for it greatly improves the quality of the end result.

Considerable peri-articular fibrosis is usually 
present when there has been a suppurative or infective arthritis and here the use of a calliper for four to six months gives good stability. Occasionally a calliper can be dispensed with altogether in these cases, particularly when the patient is light in weight.

Osteotomy is contra-indicated in elderly patients with bilateral degenerative osteo-arthritis.

\section{Excision of the Femoral Head and Neck}

The excision is performed through a SmithPetersen approach. The patient is placed on the operating table in the semi-lateral position with sandbags beneath the buttock and shoulder. The chief landmark for the incision is the anterior superior iliac spine. From this point the incision is carried posteriorly over the iliac crest for three to four inches and distally down the antero-lateral surface of the thigh for five to six inches. The interval between the Sartorius and the Tensor Fascia Femoris Muscles is defined at a point about one inch below the anterior superior spine and with the aid of retractors the incision is deepened between the Rectus Femoris medially and the Glutei laterally. Care at this stage, particularly in identifying and retracting the deepest gluteal fibres, facilitates the later stages of the operation.

The surgeon now turns to the anterior three or four inches of the iliac crest and reflects the periosteum from the lateral half of its subcutaneous surface. The gluteal attachment to the overhanging lateral margin of the crest is separated with the scalpel and then reflected sub-periosteally from the outer face of the ilium. Firm packing in the space between the muscles and the ilium controls the brisk oozing which occurs at this stage and holds the gluteal flap aside for the next stage. The tendinous origin of the Tensor Fascia Femoris Muscle from the anterior superior spine is divided and the few remaining attachments of the gluteal muscles separated from the ilium.

The distal portion of the incision in the thigh is now deepened throughout its length by dividing the fascial attachments between the Rectus Femoris medially and the Tensor Fascia Femoris and Vastus Lateralis laterally. At this stage the ascending branches of the external circumflex artery and vein should be located and divided between artery forceps where they emerge from beneath the lateral margin of the Rectus Femoris Muscle about two to three inches below the anterior superior spine.

The reflected head of the Rectus Femoris is sarefully defined, separated from its bony attachment and stripped downwards for two to three inches. The anterior surface of the capsule of the hip joint is now exposed. A longitudinal incision along the neck. of the femur, commencing at the acetabular margin and extending to the inter ${ }_{0}$ trochanteric line, is made through the joint capsulesA rougine is thrust into the cleft and the capsul levered off the superior surface of the femoral neck; a large Lane's bone lever, the curved tongue of which passes behind the neck, is there inserted. The lower border of the femoral neclos neck is exposed in the same way with the aid of $\mathbb{D}$ second bone lever. With a few touches of the knife the remaining attachments of the capsule tothe anterior margin of the acetabulum are separated and the joint is freely exposed.

Dislocation of the femoral head from the aceta $\frac{\sigma}{\sigma}$ bulum should precede section of the neck. The anterior superior margin of the acetabulum is re $\frac{3}{\mathrm{~N}}$. moved together with any osteophytes which are present, or, when the joint is fused, the ankylosis between the femoral head and acetabulum is divided with a gouge. The head is then levered out of the acetabulum by adduction and externa $\vec{b}$ rotation of the leg.

The femoral neck is now divided. The line of osteotomy commences at its inferior border as low down as possible and passes upwards anco outwards so as to divide the neck close to the greatso trochanter. If all the neck is not excised with the head, the remainder is removed with an osteotorifo care being taken to leave a smooth surface. Thes acetabulum is inspected and any osteophytes remaining round its margin are removed.

After re-attaching the reflected head of the Rectus Femoris, the wound is closed by suturing back the lateral muscle flap to the iliac crest and anterior superior spine. A few intcrrupted sutures are required between the fascia overlying the Sartorius and Tensor Fascia Femoris Muscles? No drainage is required. A firm spica bandage: applied over wool compresses the wound and by diminishing the dead space assists healing.

\section{Osteotomy}

The osteotomy can be carried out as a primary procedure at the time of the excision but preferably? as a secondary procedure three to five weeks later when the first incision has soundly healed.

When osteotomy is done primarily the distal part of the incision is extended for an inch or so, the Rectust Femoris mobilized and retracted well mediallyo and the Vastus Lateralis reflected sub-periosteallyo from the femoral shaft. With the aid of boneक levers two to three inches of the upper part of the shaft are easily exposed. The osteotomy, whicho should be cuneiform in type in order to allow? rotation of the fragments on each other without displacement, is performed just below the lesser, 


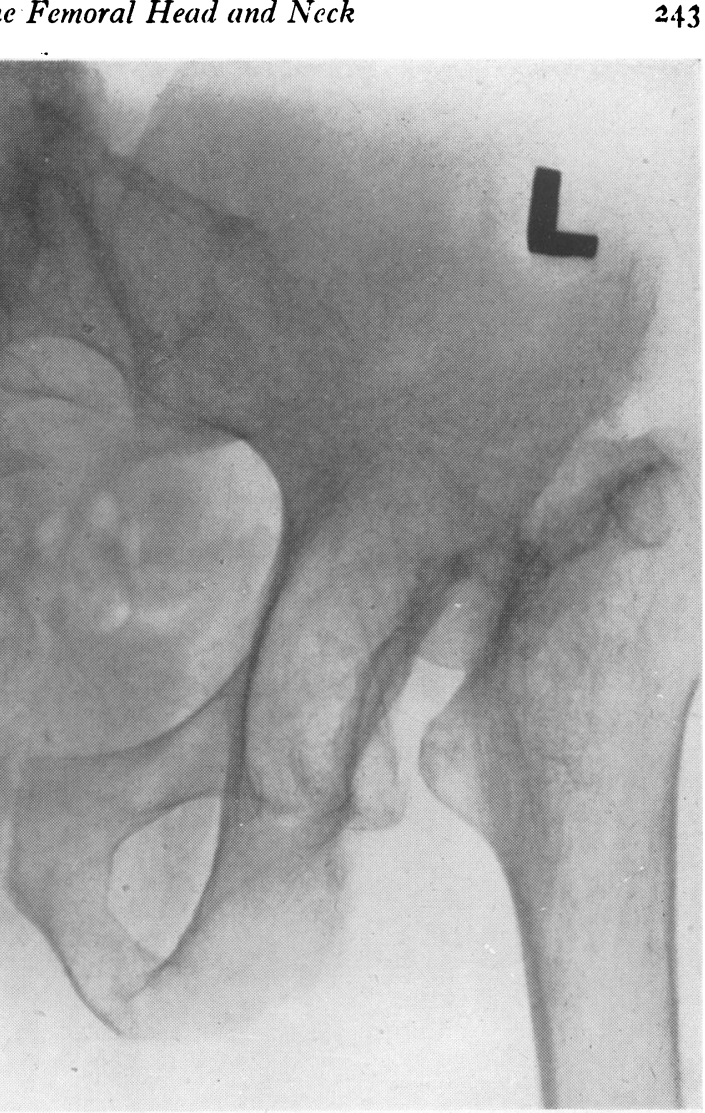

Fig. I.-Bilateral excision for ankylosing spondylitis.

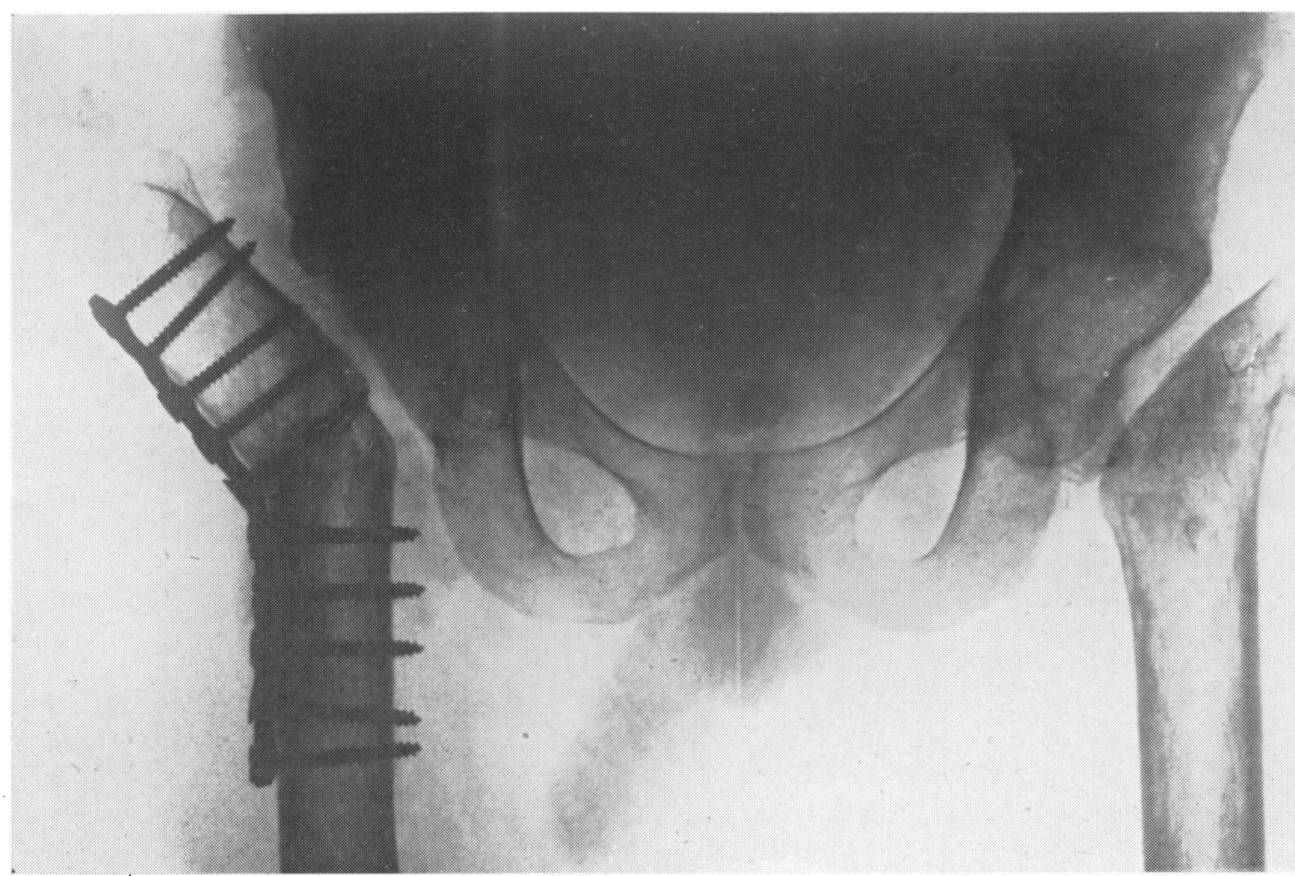

Fig. 2.-Bilateral excision and osteotomy for ankylosing spondylitis. 

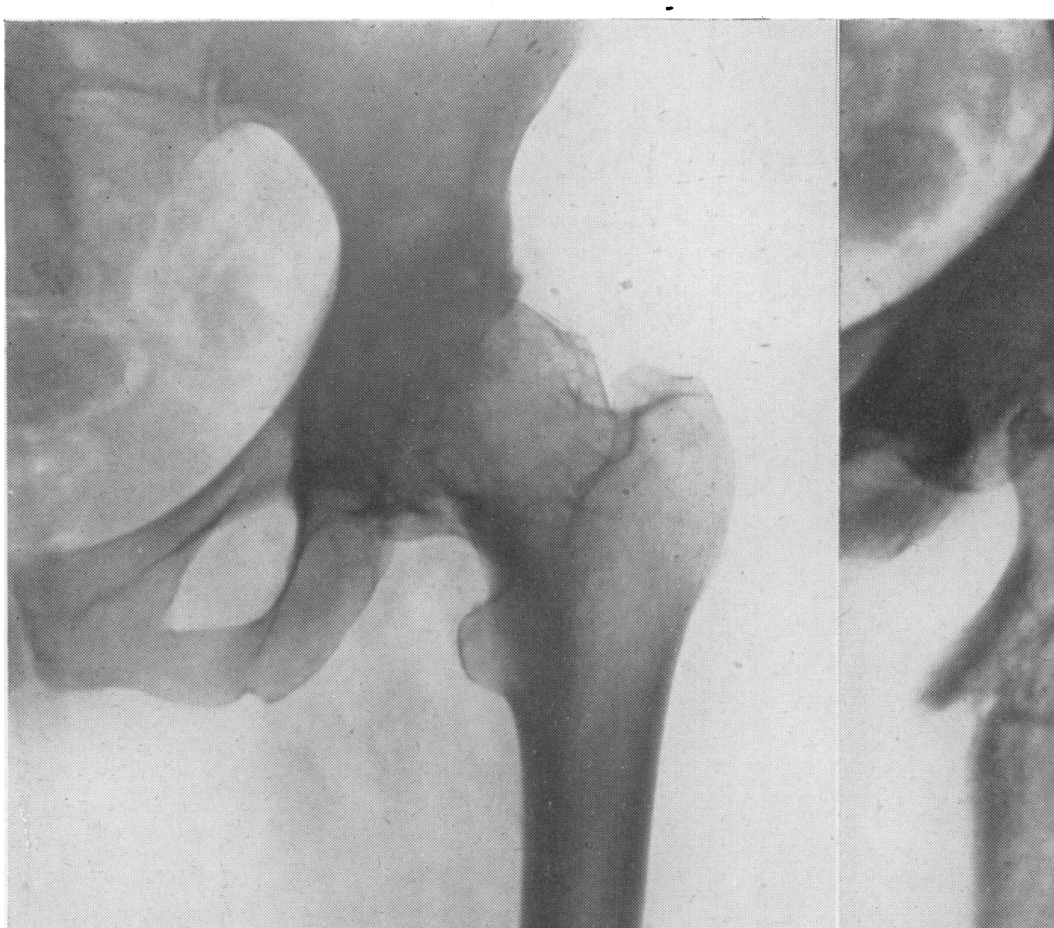

Fig. 3.-Monarticular osteo-arthritis treated by excision and osteotomy.

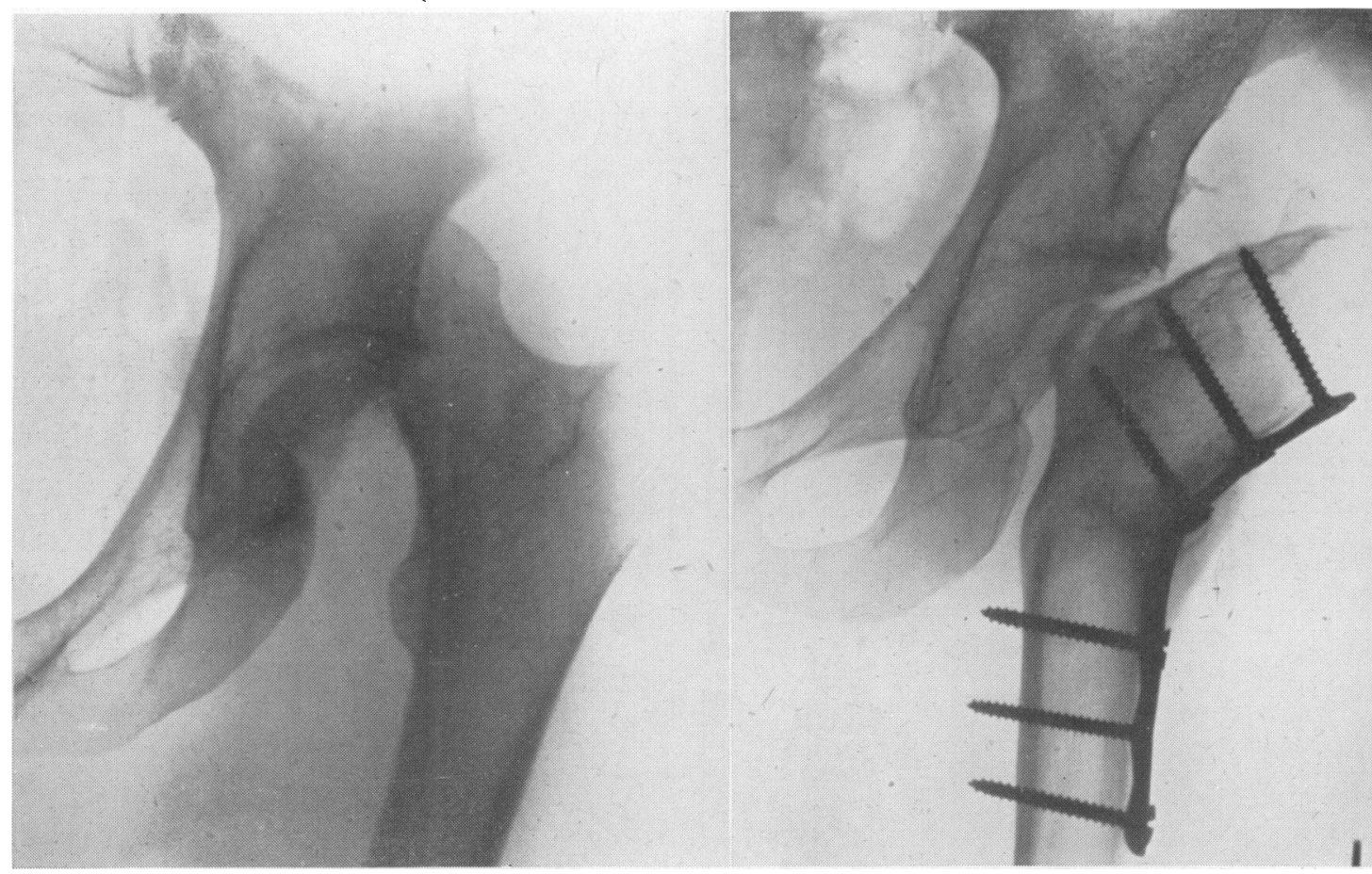

FIG. 4.-Irreducible fracture-dislocation of the hip treated by excision and osteotomy. 
FIG. 5.-Chronic suppurative arthritis treated by excision of the femoral head and neck.
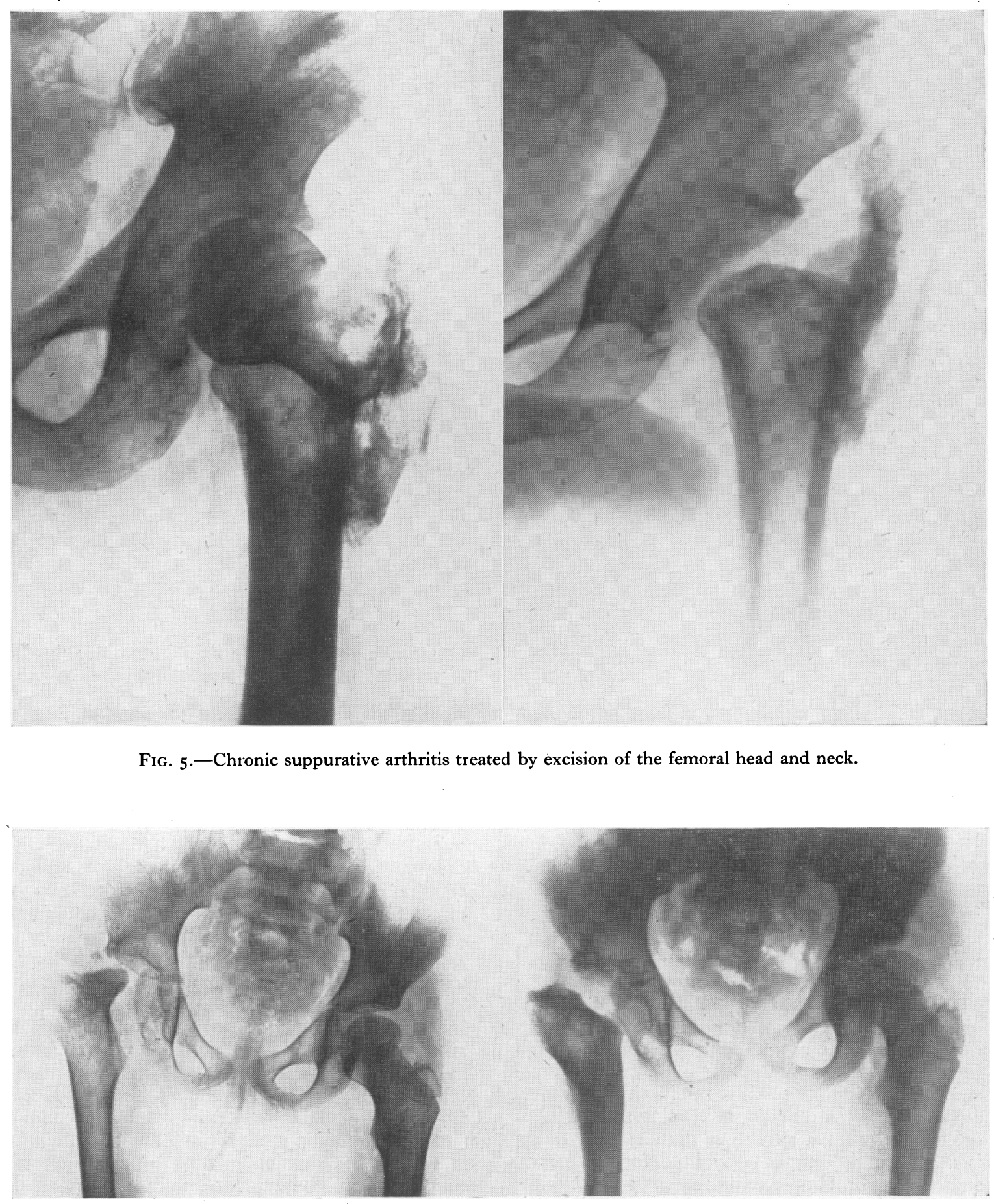

Fig. 6.-Suppurative arthritis with pathological dislocation treated by excision and osteotomy. 


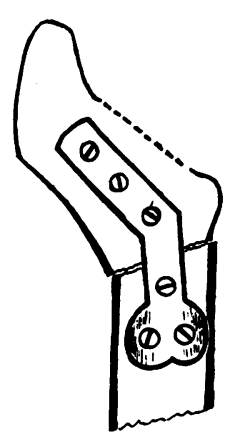

Diag. I

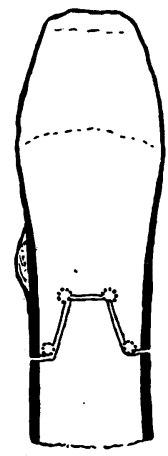

Diag. II

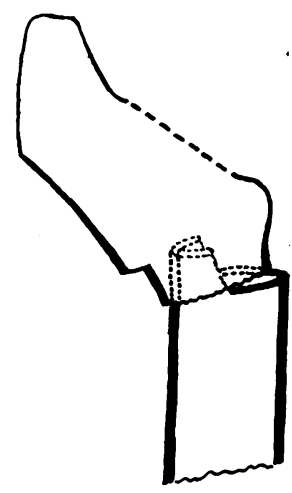

Diag. III trochanter, using small sharp osteotomes. In order to abduct the lower fragment sufficiently it is often necessary to separate a small triangular piece of bone from the lateral surface of the upper fragment (Fig. 4). The osteotomy is now fixed with a special plate angled in the coronal plane which is applied to the anterior surfaces of the great trochanter and femoral shaft (Diag. I).

When the osteotomy is done as a secondary procedure it can be performed through either a lateral or a postero-lateral approach. The use of a lateral incision entails splitting the Vastus Lateralis and with the leg abducted after the bone has been divided, thie depth of the wound makes it difficult to fix the plate in position. Technically, the osteotomy is much easier to perform through a postero-lateral approach with the patient in the prone position. An incision is made over the postcro-lateral surface of the hip, commencing over the great trochanter and extending distally for five to six inches. The fascia lata is divided lateral to the insertion of the Gluteus Maximus, thus exposing the posterior aspect of the trochanter and upper shaft. The Vastus Lateralis is reflected anteriorly fiom its attachment to the linea aspera and the upper part of the shaft and sub-trochanteric region are exposed.

The bone is divided just below the lesser trochanter, a locking type of osteotomy with a tongue of bone on the lateral aspect of the distal fragment being used in order to ensure good stability of the fragments. The tongue should be cut out first and then the bone divided around its circumference at the base of the tongue, again using small, sharp osteotomes (Diag. II). Before the distal fragment is abducted the tongue may have to be slightly shortened with bone-cutting forceps so that with the aid of a fine osteotome used as a shoe-horn it can be impacted well into the proximal fragment (Diag. III).

The osteotomy is now fixed with an eight-hole
Lane plate which may be bent to the required angle before operation. The upper fragment tends to flex after the osteotomy has been com $=$ pleted; if the patient is prone, this angulation cari be reduced by 'breaking' the table a few degrees.It is also important to guard against the tendency for the leg to fall into internal rotation while the plate is being applied. I have found that six screws give perfectly adequate grip, allowing the plate to be angled between the middle two holes which areo not used. The screws in the proximal fragmento should not penetrate the medial (weight-bearingy surface of the bone and the upper two shoutio therefore not exceed one inch in length; in the distal fragment the screws should engage th@ cortical bone medially and should be approximatelyo one and a half inches in length.

\section{Angle of the Osteotomy}

The condition of the opposite hip and lumbarō spine must be considered when estimating the correct angle of osteotomy. For unilateral osteo $\frac{0}{3}$ arthritis with a sound contralateral hip and supple lumbar spine, the distal fragment may be abducted? $40^{\circ}$ on the proximal fragment. This produces a certain amount of fixed abduction with tilting of the pelvis and apparent lengthening of the limb? In bilateral ankylosis of the hips with a supple lumbar spine, osteotomy may be performed after o excision of one hip when the contralateral hip is ankylosed by bone in adduction; the angle of the osteotomy is then adjusted to the position of the fixed hip. Osteotomy is contra-indicated when the ankylosis of the opposite hip is fibrous or when ife is fixed in abduction. When both hips are ankylosed and the lumbar spine is rigid, as in ankylosing spondylitis, an angle of osteotomy ${ }_{0}^{0}$ greater than $25^{\circ}$ may render adduction of the leg, to the neutral position impossible and should he $\frac{\text { O }}{\mathbb{Q}}$ avoided. 


\section{After-treatment for Excision and for Osteotomy}

After the operation a Steinmann's pin is inserted through the upper end of the tibia and traction applied by Hamilton-Russell extension with a weight of $7 \mathrm{lb}$. The chief advantage of the tibial pin is that it controls rotation and prevents eversion of the limb, which can be troublesome after this operation. It is important that shortening should be prevented and that the great trochanter should lie below the level of the acetabulum. The position of the hip should be checked by X-ray examination the day after operation. Shortening with upward displacement of the great trochanter on to the dorsum ilii will of course lead to troublesome instability.

After fourteen days movements for the hip and knee and exercises for the thigh and hip muscles are commenced. Joint movements are at first performed through a limited range with the extension apparatus in place. After four weeks the weight is removed during treatment so that a free range of movement at the hip and knee can be obtained,

In a straightforward case of excision without osteotomy, traction is continued for eight weeks. The patient then commences walking with the aid of crutches or a walking-machine and with a weight-relieving calliper, the measurements for which were taken during the period of traction. The calliper is worn for four to eight months and when clinical tests indicate that the hip is stable is gradually dispensed with.

When osteotomy has been performed traction is maintained for ten to twelve weeks. Weight bearing without a calliper is commenced when X-ray examination shows that the osteotomy has united.

\section{Discussion and Results}

\section{Ankylosing Spondylitis}

Here a free range of movement at both hips is essential ; otherwise the fixed spine makes it impossible for the patient to sit in comfort. In assessing the results the severe degree of crippling frequently found in many of these patients must be appreciated ; some of them have been bedridden for years with ankylosis of the hips, knees and spine. The prospect of a good functional result is brighter when the knees are unaffected, for ankylosis of these joints makes sitting difficult even when the hips move freely. After the surgical treatment has been completed a prolonged and laborious course of 'training extending over two years or more is essential to achieve the optimal degree of functional recovery. This necessitates considerable co-operation on the part of the patient.
Of the seven cases treated, excision of the head and neck of both hips has been performed in six, followed in four cases by osteotomy. My first case in this group, a man aged thirty-five whose treatment was commenced in 1938 , was one of the two in whom bilateral excision only was performed. There was an interval of about seven months between the two operations and after each operation the patient used a weight-relieving calliper for six months. The result has been most satisfactory, for he now has a pair of relatively painless and stable hips with a range of flexion of $80^{\circ}$ (Fig. I). He can walk moderate distances using sticks and drives a car. The second patient in whom bilateral excision was performed walks with the aid of elbow crutches but the functional result is marred by ankylosis of koth knees.

Of the four cases in which excision followed by osteotomy was performed on both hips, good stability has been secured without the use of callipers (Fig. 2). These four patients have a good range of movement at the hips and can walk with the aid of sticks or elbow crutches. The patient in whom the excision of only one hip was performed, when last examined, could walk reasonably well. It was intended to proceed with excision of the other hip but the war interrupted his treatment and he has been lost sight of.

\section{Traumatic and Degenerative Osteo-Arthritis}

In six patients with bilateral degenerative osteoarthritis of the hips, excision of the head and neck has been performed on the more painful hip. Osteotomy is not indicated in these cases, for the tilting of the pelvis which it produces would throw an adduction strain on the contra-lateral hip. The results in this group of patients have been disappointing. Although the operation has relieved pain and restored a free range of movement in one hip, function is poor, for the gait in the majority of these patients is slow and halting. In bilateral osteo-arthritis of the hips, therefore, excision of one hip is indicated only when pain is severe; a good functional recovery cannot be expected.

Excision followed by osteotomy has been carried out in six patients with unilateral osteo-arthritis. The need for osteotomy in this group was indicated by the progress made by my first patient with a unilateral osteo-arthritis after he had been treated by this method (Fig. 3). After excision of the femoral head and neck in 194I a weight-relieving calliper was worn for eight months. When the calliper was discarded the hip felt unstable and ached after use. These symptoms were completely relieved by a sub-trochanteric osteotomy (Fig. 4).

In five patients in this group the results are very satisfactory. The range of flexion varies from $75^{\circ}$ 
to $90^{\circ}$, the hips are painless and stable and the gait is good with only a slight limp. One elderly patient refused to co-operate in carrying out aftertreatment and did not learn to walk.

\section{3. (a) Fracture-dislocation of the Hip}

Excision of the femoral head and neck followed by osteotomy has been performed in three cases of irreducible fracture-dislocation of the hip (Fig. 4).

\section{(b) Ununited Fracture of the Femoral Neck}

In this condition there is a tendency to advise some form of osteotomy almost as a routine. A good result may be expected if the osteotomy is followed by union of the fracture but unfortunately, owing to degenerative changes in the femoral head and absorption of the neck, the fracture fails to unite in a high proportion of cases. The patient is then left with a stiff, painful and often unstable hip. I have therefore excised the femoral head and remainder of the neck in four patients with ununited fracture of the femoral neck. In three cases the excision was followed by an osteotomy.

In these two groups the results, like those in patients with degenerative and traumatic osteoarthritis, are with one exception most satisfactory. Of the seven patients, six have a good range of movement, are free of pain and walk well. In the one case of ununited fracture of the femoral neck in which excision of the head was not followed by osteotomy, the result has been unsatisfactory owing to shortening and adduction deformity.

\section{Bilateral Ankylosis from Suppurative Arthritis}

Four such cases have been treated. In two good results were obtained by excision without osteotomy, for excellent stability was provided by fibrosis around the joint. In one case the excision was followed by an osteotomy and here the result was only fair owing to limitation of flexion $\left(40^{\circ}\right)$. In the fourth patient, on whom arthroplasty had been attempted elsewhere, a range of flexion of only $30^{\circ}$ has been obtained after excision of the head and neck.

\section{Chronic Suppurative Arthritis}

In this group there are four patients, in three of whom long-standing infection with multiplo sinuses had persisted despite numerous operations for drainage and sequestrectomy. Here the in fection appears to linger in the relatively avasculaep cancellous bone of the femoral head and it mas prove impossible to obtain sound healing until the femoral head has been removed. In all of these cases excision of the femoral head and neck re $\mathbb{\mathbb { Q }}$ moved the main site of infection and allowed the्छ sinuses to heal (Fig. 5).

In the three cases with long-standing infection: adequate stability was provided by periarticulaw fibrosis ; in the fourth case, a child of nine with 옹 recent infection and pathological dislocation, afs osteotomy was performed (Fig. 6). The results obtained in this group have been very satisfactory + Although the range of flexion is somewhat limited the hips are stable and painless.

\section{Summary}

I. In unilateral traumatic and degenerative osteo-arthritis, ununited fracture of the femora neck and fracture-dislocation of the hip, excision of the femoral head and neck followed by ostenco tomy can be relied upon to give a painless stable joint with an average range of flexion $75^{\circ}$ to $90^{\circ}$.

2. In ankylosing spondylitis and rheumatoi arthritis, excision of both hips followed by osteoڤึ tomy has restored to limited activity patients whœ were previously bedridden.

3. In bilateral ankylosis of the hips following in fection, excision of one hip followed by the use of a calliper has produced a stable joint with an aver: age range of flexion of $60^{\circ}$.

4. In four patients with chronic suppurativè arthritis, three of whom had discharging sinuses, excision of the femoral head and neck was followe $\$$ by healing of the sinuses and restoration of limited range of painless movement. 